\title{
DYNAMIC SITE LAYOUT PLANNING USING MTPE PRINCIPLE FROM PHYSICS
}

\author{
Mohsen Andayesh* and Farnaz Sadeghpour \\ Department of Civil Engineering, University of Calgary, Calgary, Canada \\ *Corresponding author (m.andayesh@ucalgary.ca)
}

\begin{abstract}
Site layout planning is the task of determining optimum location for construction facilities on the site in order to increase productivity and safety of the project. Most of the existing studies assume all facilities are required for the entire duration of the project which is not a realistic scenario. More advanced studies have proposed dynamic models to reflect the changes in the project requirements over the course of time. They generate optimum layouts for a set of consecutive time intervals. However, combining individually optimized layouts does not necessarily guarantee globally optimum locations for facilities. In addition, forcing facilities to fit in a set of predetermined time intervals can lead to waste of space and inefficient layouts.

This research adopts the Minimum Total Potential Energy (MTPE) principle from physics to develop a dynamic planning model which generates layouts that are globally optimized. It assumes construction site to be a physical system in which construction facilities represent particles and the internal forces among particles reflect the closeness constraints between facilities. The total potential energy of the system represents the fitness of the layout for the defined objective function. Based on MTPE principle, the internal forces cause particle to search for a location with lower potential energy until the system reaches equilibrium. The final state of particles represents the minimum total potential energy of the system, which reflects the fittest arrangement for the facilities on the site. A computational example is analyzed to investigate the accuracy of the proposed model and demonstrate its capabilities.
\end{abstract}

Keywords: Dynamic Site Layout Planning, Construction Site Safety, Construction Site Management, Optimization

\section{INTRODUCTION \& BACKGROUND}

Site space is considered a limited resource beside materials, equipment, labor, time, and money [1]. The importance of site layout planning and its impact on the productivity, safety, and cost of the project has been emphasized repeatedly in the literature ([2], [3]). The main concern in site layout planning is determining the optimum location of construction objects such as temporary facilities (e.g., management office, geotechnical laboratory), major equipment (e.g., tower crane), and material storage areas (e.g., gravel depot area) on the site considering cost, safety and security issues. Several site layout models have been developed using different techniques and methods such as genetic algorithms (e.g., [4], [5]), knowledge-based systems (e.g., [1], [3]), geometric reasoning (e.g., [2], [6]), and metaheuristic methods (e.g., [7]). Regardless of the utilized method, site layout models could be classified into two general categories based on how they model the time factor and reflect the changes that occur in the site during the project: static models, and dynamic models.

In static models, all construction objects are assumed to exist on the site for the entire duration of the project without any changes. In reality, objects are required for limited periods of time depending on activities they are associated with. As an example, Fig. 1 shows the schedule for nine objects which are required for different periods of time. Static models do not reflect the changes in space requirements in the site, and as a result, they do not allow for the reuse of space. For instance, although objects 1 and 8 in Fig. 1 do not have any time overlap, in a static model they are not allowed to use the same space on the site. To overcome this limitation, dynamic models aim to reflect 
the changes in construction requirements and make more efficient use of space. To reflect the changes over the time, some models divide the project duration in several time intervals and generate an optimum partial layout for each. This provides the possibility of reusing space from one partial layout to another. For example in Fig. 1, the schedule is divided into two time intervals. Some objects in the first partial layout are no longer required for the second partial layout (objects 1, 2, and 7) and therefore, their occupied space would be available for objects in second partial layout (objects 5, 8, and 9). There are also three objects that exist in both layouts (objects 3, 4, and 6). Therefore, there are six objects that need to be located in each partial layout. This will increases their chance of getting more optimum locations compared with the static scenario where nine objects have to be located in the same space.

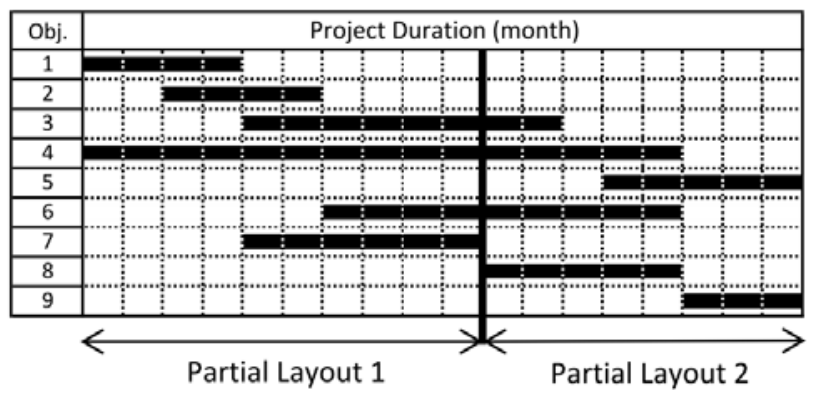

Fig. 1 Duration of construction objects on the site

Despite the above-mentioned advantage, most of the models which divide the project duration in time intervals, optimize partial layouts separately and in chronological order (e.g., [4], [8]). In such cases, the locations of objects in the future partial layouts are highly influenced by those in early layouts [9]. In addition, combining several individually optimized partial layouts does not necessary lead to an overall optimum layout. Further, although partial layouts offer more efficient use of space over static models (e.g. locating 6 objects as opposed to 9), they still do not present the most efficient use of space. For instance, in the example of Fig. 1, only four objects are required on the site at any given time while in each partial layout, six objects are assumed to exist during each time interval. This does not represent a realistic need of space on the site and therefore, decreases the chance of objects for getting more optimum locations. This paper presents a dynamic site layout model to overcome the limitation mentioned above.

\section{A DYNAMIC MODEL FOR LAYOUT PLANNING}

The model presented in this paper determines the global optimum location of objects in the site considering the actual project requirements in time. In this model time is considered a continuous quantity and objects exist on the site for the actual period of time they are required for. Accordingly, objects which do not have time overlap with one another (e.g. objects $1,7,8,9$ ) can occupy the same space on the site. This enables the model to reuse the space of objects which are no longer required on the site. The model starts from a random initial arrangement of objects in the site and improves the layout gradually using Minimum Total Potential Energy (MTPE) principle from the physics. All objects involved in the project (e.g. all nine objects in Fig. 1) will search for their optimum locations simultaneously, although their space allocation will be based on the time they exist on the site. As a result, objects in the later periods of time (e.g. object 8) will have the same chance as early objects (e.g. object 1 ) to get optimum locations. Objects with higher level of importance will beget precedence over others by the weight assigned to them. As a result, objects with higher impact on the overall fitness of the layout can claim and get better locations even if that position is occupied by other objects. The next section will illustrate the details of the developed model.

\section{MODEL DEVELOPMENT}

\subsection{Objective function}

In construction sites, resources such as material, labor, and equipment travel between objects to perform or support construction activities. For instance gravel should be moved from its storage area to the batch plant, and the prepared concrete should be delivered to the structure under construction to be used. There is a cost associated with such resource exchanges between two locations. The developed model aims to minimize the total for this travel cost on the site. This cost is a function of the mutual workflow and distance between objects which can be written as:

objective function $=\sum W_{i j} d_{i j}$ 
where $\mathrm{W}_{\mathrm{ij}}$ reflects the closeness weight between facilities $\mathrm{i}$ and $\mathrm{j}$, and $\mathrm{d}_{\mathrm{ij}}$ represents the distance between object $\mathrm{i}$ and $\mathrm{j}$ ([2], [6]). The closeness weights can be defined based on the actual cost of workflow between two objects (e.g. the cost of moving concrete from batch plant to the structure). This workflow is commonly reflected by proximity closeness weights between objects (e.g., [4], [8]). In such cases, relatively large closeness weights indicate significant workflow cost between objects and accordingly, the two objects will be have to be close to one another in order to minimize the objective function.

\subsection{Minimum Total Potential Energy (MTPE) principle for site layout planning}

The model presented in this paper determines the optimum location of objects using the energy principles governing a physical system of particles. In this system, the potential energy depends on the internal forces and distances between particles. For instance, in a system composed of a ball and the earth, the potential energy is a function of the ball's weight and its distance from the earth. The total potential energy in a system of particles is presented as:

$P E=\sum F_{i j} d_{i j}$

where $F_{i j}$ represents the force between particles $i$ and $j$, and $\mathrm{d}_{\mathrm{ij}}$ shows the distance between the two particles. The internal forces acting on particles cause them to move and gain kinetic energy. According to the law of conservation of energy, the total energy (i.e. potential energy + kinetic energy) in a system remains constant [9]. As a result, when the particles gain kinetic energy, the system looses part of its potential energy. If the kinetic energy is wasted immediately after the particle moves (for instance due to friction), it will not transfer back to potential energy. Under such circumstances, the total potential energy decreases gradually until the system reaches equilibrium state where all the forces are balanced and particles no longer move. The equilibrium state represents the minimum total potential energy condition of the system.

In the developed site layout model, the objects are assumed as particles, and the closeness weights are reflected as pulling forces between the particles. Accordingly, the potential energy of the system of particles (Eq. 2) reflects the objective function of the site layout (Eq. 1). The closeness weights (internal forces) cause objects (particles) to move in search for an equilibrium state. As the objects move, the objective function (potential energy) decreases until the layout (system) reaches equilibrium. In this state, the objective function is at its minimum value and the locations of objects present the optimum layout.

\subsection{Modeling construction objects}

In site layout planning, construction objects are classified in two categories based on how they are located on the site: fixed objects and positionable objects. Fixed objects, such as structures under construction, have a known location prior to construction and they are assumed to be fixed in their location. On the other hand, positionable objects are those that have to be located during the site planning process. Examples for these objects include tower cranes, material lay down areas, and management offices. Construction objects can exist on the site for the entire duration of the project or a limited period of time.

In this model construction objects are represented by their minimum bounding circles. Circular shape of objects facilitates their move as they search for optimum locations. During the search, objects might clash with site boundaries and other objects. The curved shape of circles allows the objects to move smoothly around each other when they clash and not get trapped in angular corners. This will be explained in more details in section 3.5.

\subsection{Search for the optimum position}

In this model, the closeness weight between two objects (e.g. $\mathrm{O} 1$ and $\mathrm{O} 4$ in Fig. 3) is defined as two equal forces that act on the particles and pull them toward each other in a physical system (e.g. $F_{1-4}$ and $F_{4-1}$ ). For instance, in Fig. 2 three forces are acting on a positionable object $\mathrm{O}_{4}$, representing three closeness weights that $\mathrm{O}_{4}$ has with three fixed objects: $\mathrm{O}_{1}, \mathrm{O}_{2}$, and $\mathrm{O}_{3}$. The forces acting on an object cause it to move toward its resultant force, which is the vector summation of all forces acting on the object (R) (see Fig. 2a). Since the closeness weights are constant, the magnitudes of their respective internal forces will also remain constant. However, changes in the position of the objects will cause the direction of acting forces to change. 
An object will continue to move toward the new direction of its resultant force until all acting forces cancel one another and the object reaches equilibrium (Fig. 2b). In this state, the acting forces are balanced, the resultant force equals zero, and accordingly, $\mathrm{O}_{4}$ no longer moves.

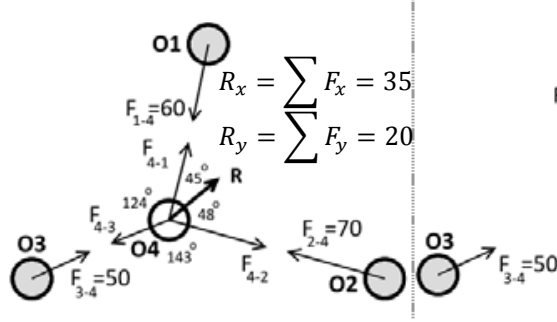

(a)

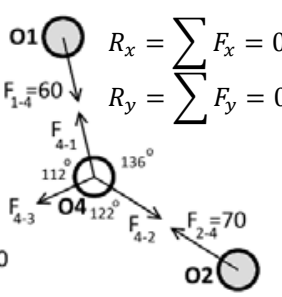

(b)
Fig. 2 Acting forces on an object: a. moving state; b. equilibrium state

Here, the speed -as it applies to particles in a physical system- is ignored, i.e. the forces acting on objects cause them to move and change their position with zero velocity $(\mathrm{v}=0)$. This assumption has a significance to enable the objects to reach the equilibrium state. It causes the kinetic energy to vanish $\left(1 / 2 m v^{2}=0\right)$ and not transfer back to potential energy. Also by this assumption, the objects will not get momentum while they move to search for an optimum location $(m v=0)$. Therefore, they will not bounce when they clash with site boundaries or other objects; instead they will smoothly slide by each other. By eliminating speed, the objects will move toward their resultant force, which directs them towards their optimum location, at any given time. If the objects are allowed to gain speed, they will not necessary move toward their resultant force. Further, when objects reach the intended optimum location, they would keep moving since although their resultant force is zero but the objects have speed. Therefore in order to reach the desired equilibrium state, it is important to assume there is no speed in the system.

\subsection{Clash detection}

While the objects search for the equilibrium state, they might clash with site boundaries or other objects. An object clashes into the site boundary when its resultant force pulls it toward the boundary (Fig. 3a). In this case, the boundary returns a reaction force (RF) to the object which is equal to the perpendicular component $\left(\mathrm{R}_{\mathrm{p}}\right)$ of the resultant force in magnitude but in the opposite direction $\left(|\overrightarrow{R F}|=\left|\overrightarrow{R_{p}}\right|\right)$. When the reaction force acts on the object, it cancels $R_{p}$, and as a result, the tangent component $\left(\mathrm{R}_{\mathrm{t}}\right)$ of the resultant force will be the only one that moves the object. The same scenario happens when a positionable object clashes with a fixed object. Since the fixed object is steady in its position, it returns a reaction force (RF) equal to the perpendicular component of the resultant force $\left(\mathrm{R}_{\mathrm{p}}\right)$ to the positionable object which causes the positionable object to slide along the fixed object (Fig. 3b).

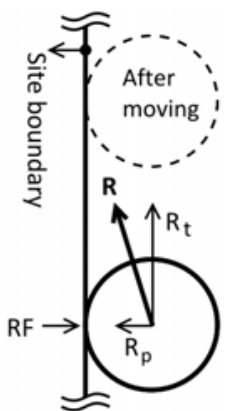

(a)

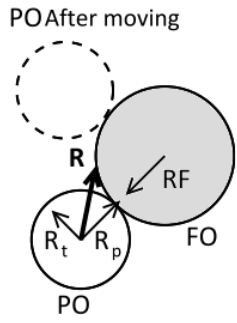

(b)

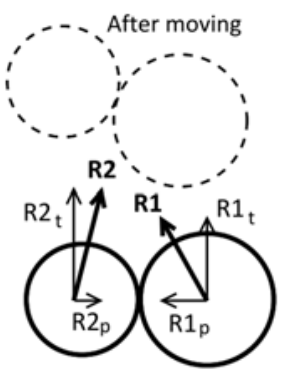

(c)
Fig. 3 Possible clashes: a. object-boundary, b. positionable object (PO) - fixed object (FO), and c. two POs

When two positionable objects clash with one another, they start to push each other and try to continue moving in the direction of their resultant force (Fig. 3c). Eventually the object with stronger perpendicular force $\left(R 1_{p}\right.$ or $\left.R 2_{p}\right)$ will be able to push the other object. In this condition, each object will move based on the vector summation of the tangent component of its resultant force $\left(R 1_{t}\right.$, or $\left.R 2_{t}\right)$ and half of the difference between two perpendicular components $\left[\frac{1}{2}\left(R 1_{p}-R 2_{p}\right)\right]$. As the locations of two objects change, the resultant forces, and as a result, the perpendicular and tangent components will change until both objects reach equilibrium. Objects with larger forces are able to push other objects and can get their desired location even if another object has occupied that position. This means objects with higher closeness weights will get better locations regardless of their arrival time or initial location in the search.

\subsection{Search process}

The search for optimum layout starts from a random initial distribution of all the objects that exist in the different time 
periods of the project. As will be discussed later, the initial location of objects will not have an impact on the final layout. All positionable objects will move simultaneously (based on their internal forces defined by closeness weights) to search for their optimum locations. As objects move in the direction of their resultant force, the objective function of the layout will decrease continuously (similar to potential energy of a system). The model considers the time associated with each object in this search. Therefore, objects that exist in different time periods can pass over each other or stop in the space occupied by others. This means that in the proposed model, the space occupied by objects can be reused for other objects in the previous or future periods of time. When objects belonging to the same time periods clash with one another, they will start to push each other and compete over better locations as described in 3.5. This enables objects with higher closeness weights to push other objects and get their desired location regardless of their arrival time on the site or their initial location. The objects will continue to move and the objective function will decrease continuously until the system reaches equilibrium, i.e. all forces acting on every individual object are balanced. At this equilibrium state, the objective function reaches its minimum value and the most optimum layout has reached. Therefore, the final layout generated by this model represents the global optimum arrangement of objects over the time.

\section{COMPUTATIONAL EXAMPLE}

Assume the example of a construction site with three fixed objects (FO1, FO2, and FO3) and five positionable objects (PO1 to PO5). Fig. 4 presents a schematic of the site shape, the position of the fixed objects, and the initial location for the positionable objects. The total duration of the project is assumed to be 10 months but objects are required for different periods of time. Table 1 summarizes the properties of the construction objects and Table 2 presents the closeness weights between them.

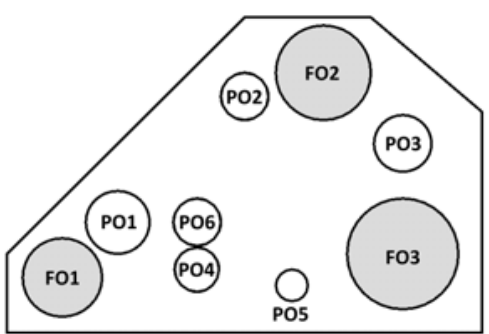

Fig. 4 Initial arrangement of objects in the site

Table 1. Properties of the construction objects

\begin{tabular}{lcccc}
\hline Objects & Type & $\begin{array}{c}\text { Radius } \\
(\mathrm{m})\end{array}$ & $\begin{array}{c}\text { Existing time } \\
(\text { months })\end{array}$ & $\begin{array}{c}\text { Center } \\
\text { Position }^{\text {a }}\end{array}$ \\
\hline FO1 & Fixed & 25 & $1-10$ & $(35,35)$ \\
FO2 & Fixed & 30 & $1-10$ & $(200,165)$ \\
FO3 & Fixed & 35 & $2-9$ & $(250,50)$ \\
\hline PO1 & Positionable & 20 & $1-10$ & $(70,70)$ \\
PO2 & Positionable & 15 & $2-4$ & $(150,150)$ \\
PO3 & Positionable & 18 & $5-8$ & $(250,120)$ \\
PO4 & Positionable & 14 & $4-8$ & $(120,40)$ \\
PO5 & Positionable & 10 & $4-10$ & $(180,30)$ \\
PO6 & Positionable & 15 & $3-8$ & $(120,70)$
\end{tabular}

${ }^{\mathrm{a}}$ For POs this represents the initial location

Table 2. Closeness weights between objects

\begin{tabular}{lcccccc} 
Objects & PO1 & PO2 & PO3 & PO4 & PO5 & PO6 \\
\hline FO1 & 40 & 170 & 115 & 100 & 0 & 70 \\
FO2 & 0 & 120 & 90 & 60 & 70 & 30 \\
FO3 & 90 & 100 & 80 & 80 & 50 & 0 \\
\hline PO1 & - & 0 & 0 & 0 & 10 & 20 \\
PO2 & - & - & 0 & 0 & 0 & 0 \\
PO3 & - & - & - & 10 & 0 & 0 \\
PO4 & - & - & - & - & 0 & 8 \\
PO5 & - & - & - & - & - & 15 \\
PO6 & - & - & - & - & - & - \\
\hline
\end{tabular}

A prototype tool has been developed using Java programming language to implement the described model. When the optimization starts, the objective function decreases as the objects move toward their equilibrium state (see Fig. 5). The model continues the search process until no change is observed in the objective function. It took less than a second (35 milliseconds) for the model to reach the optimum layout (presented in Fig. 6) for this example on a regular office computer $(2 \mathrm{GHz}$ Core $2 \mathrm{Duo}$ CPU, 3MB cache memory, and 3 GB of RAM). 


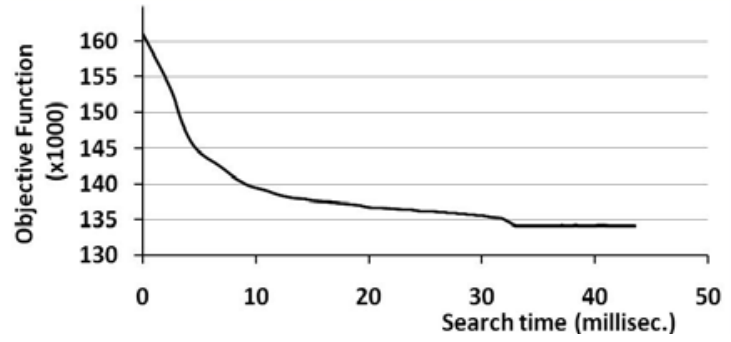

Fig. 5 Change in the objective function value as the layout improves

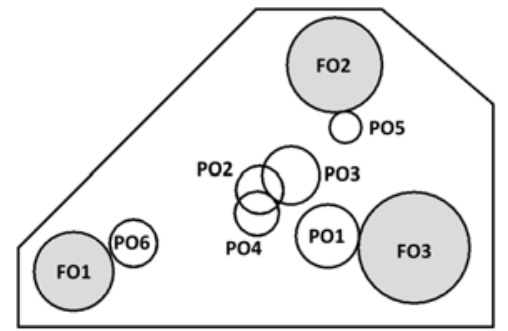

Fig. 6 Optimized final layout for the example

In the final layout shown in Fig. 6, it might appear that PO2 is overlapped with PO3 and PO4. In reality, this is not an actual overlap and does not result in space conflict since PO2 does not have any time overlap with PO3 and PO4 (see Table 1). In fact, the model is reusing the space occupied by PO2 during months 2 to 4 for PO3 and PO4 in later stages of the project (months 5 to 8 , and 4 to 8 , respectively). It can also be noticed that in the final layout PO3 is adjacent with PO4. This means that these two objects where competing over the same space. Although PO4 enters the site sooner than PO3, i.e. month 4 versus month 5 (see Table 1), PO3 has a better chance to get its desired space due to its larger closeness weights (see Table 2), which is an indication of its importance over PO4.

\section{CONCLUDING REMARKS}

This paper presented a dynamic layout planning model that is capable to generate layouts that are optimized over the duration of the project. The optimization process is modeled after Minimum Total Potential Energy principle from the physics. Based on this principle, the closeness weights (internal forces) cause the objects (particles) to move from their random initial location in search for the equilibrium state. This state has the global minimum objective function (potential energy). Objects from different time periods of the project start the search simultaneously. This means all objects will have the same chance to get optimum location regardless of the order they arrive to the site. However, objects occupy space only for the actual duration they are required for, and their space will become available for objects in the previous or future periods of time. In addition, in the presented model, the final location of objects does not depend on their initial location. Objects with higher influence on the overall fitness of the layout are able to get their desired location even if initially it has been occupied by other objects. The presented model is the first to develop a global optimum in dynamic site layout modeling.

\section{REFERENCES}

[1] Tommelein, I. D., and Zouein, P. P., "Interactive Dynamic Layout Planning," J. Constr. Eng. Manage., 119(2), pp. 266-287, 1993.

[2] Sadeghpour, F., Moselhi, O., and Alkass, S., "A CADbased model for site planning," Autom. Constr., 13(6), pp. 701- 715, 2004.

[3] Tommelein, I. D., Levitt, R. E., and Hayes-Roth, B., "SightPlan Model for Site Layout," J. Constr. Eng. Manage., 118(4), pp. 749-766, 1992.

[4] Elbeltagi, E., Hegazy, T., Hosny, and Eldosouky, A., "Schedule-dependent evolution of site layout planning," Constr. Manage and Economics, 19(7), pp. 689-697, 2001.

[5] Li, H. and Love, P. E. D., "Site-Level Facilities Layout Using Genetic Algorithms," J. Comput. Civ. Eng., 12(4), pp. 227-231, 1998.

[6] Sadeghpour, F., Moselhi, O., and Alkass, S., "Computer-Aided Site Layout Planning," J. Constr. Eng. Manage., 132(2), pp. 143-151, 2006.

[7] H. Zhang and J. Y. Wang, "Particle Swarm Optimization for Construction Site Unequal-Area Layout," J. Constr. Eng. Manage., 134(9), pp. 739-748, 2008.

[8] P. P. Zouein and I. D. Tommelein, "Dynamic Layout Planning Using a Hybrid Incremental Solution Method," $J$. Constr. Eng. Manage., 125(6), pp. 400-408, 1999.

[9] K. El-Rayes and H. Said, "Dynamic Site Layout Planning Using Approximate Dynamic Programming," $J$. Comput. Civ. Eng., 23(2), pp. 119-127, 2009.

[10] Raymond A. Serway and John W. Jewett, Physics for Scientists and Engineers, Brooks/Cole, 8th ed., 2010. 